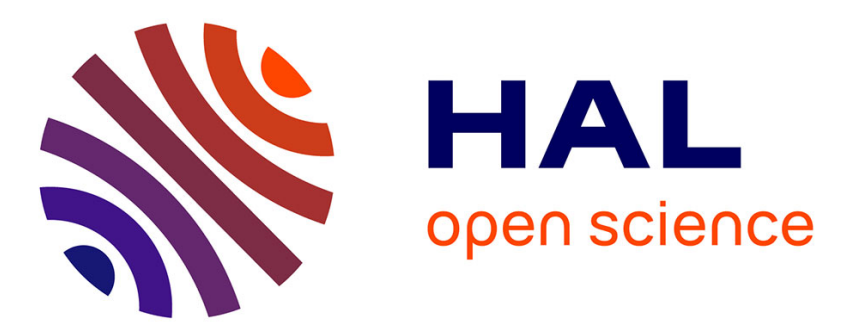

\title{
Computational modeling of thoracic and abdominal anatomy using spatial relationships for image segmentation
}

Oscar Camara, Olivier Colliot, Isabelle Bloch

\section{- To cite this version:}

Oscar Camara, Olivier Colliot, Isabelle Bloch. Computational modeling of thoracic and abdominal anatomy using spatial relationships for image segmentation. Real Time Imaging, 2004, 10 (4), pp.263273. 10.1016/j.rti.2004.05.005 . hal-01251241

\author{
HAL Id: hal-01251241 \\ https://hal.inria.fr/hal-01251241
}

Submitted on 5 Jan 2016

HAL is a multi-disciplinary open access archive for the deposit and dissemination of scientific research documents, whether they are published or not. The documents may come from teaching and research institutions in France or abroad, or from public or private research centers.
L'archive ouverte pluridisciplinaire HAL, est destinée au dépôt et à la diffusion de documents scientifiques de niveau recherche, publiés ou non, émanant des établissements d'enseignement et de recherche français ou étrangers, des laboratoires publics ou privés. 


\title{
Computational modeling of thoracic and abdominal anatomy using spatial relationships for image segmentation
}

\author{
O. Camara * ${ }^{*}$ O. Colliot ${ }^{\dagger}$ and I. Bloch \\ ENST Paris, \\ TSI Department, CNRS UMR 5141 LTCI, \\ 46, rue Barrault, 75634 Paris Cedex 13, France \\ \{Oscar.Camara, Olivier.Colliot, Isabelle.Bloch\}@enst.fr
}

This paper presents an original hierarchical segmentation approach of several thoracic and abdominal structures in $\mathrm{CT}$ and emission PET images. Segmentation results will be used to initialize a non-linear registration procedure between these complementary imaging modalities. Therefore, structures involved in the segmentation system must be visible in both CT and emission PET images in order to compute a spatial transformation between them. Thus, the chosen structures include lungs, kidneys and liver (skin and skeleton are also segmented as support structures). In the hierarchical segmentation procedure, the extraction of a given structure is driven by information derived from a simpler one. This information is composed of spatial constraints inferred from the previously segmented structures and expressed by means of Regions Of Interest (ROI) in which the search for new structures will take place. The segmentation of each structure follows a two-phase process: a first stage is composed of automatic thresholding and other low level operations in the ROI defined by previously segmented objects; a second stage employs a 3D deformable model to refine and regularize results provided by the former step. Visual inspection by medical experts has stated that the proposed segmentation approach provides results which are accurate enough to guide a subsequent non-linear registration procedure.

\section{Introduction}

Segmentation or recognition of anatomical structures in medical images is at the core of current state-of-the-art research in image processing and computer vision fields. The constant improvement of the quality of these images and the availability of powerful computers allow to develop medical segmentation-based procedures (atlas construction, medical training) helping physician's critical decisions. In addition, structure recognition is frequently used as a first step of other image processing techniques such as registration. Nevertheless, medical image segmentation remains a challenging task due

\footnotetext{
*O. Camara's current address: Computational Imaging Science Group, Radiological Sciences, Guy's Hospital, King's College London, London SE1 9RT, UK, Oscar.Camara@kcl.ac.uk

†O. Colliot's current address: McConnell Brain Imaging Center, Montréal Neurological Institute, McGill University, Montréal, Québec, H3A2B4, Canada, colliot@bic.mni.mcgill.ca
}

to either inter-patient variability or imperfections linked to image acquisition devices (noise, lack of contrast, weak contours).

Oncology is the branch of medicine that studies cancer disease. In this domain, physicians use images acquired by two complementary imaging modalities: Computed Tomography (CT) and Positron Emission Tomography (PET). CT images have good SNR (Signal to Noise Ratio) quality and spatial resolution, thus giving an accurate anatomical information about visualized structures. But, they do not provide any functional or metabolic information, which is crucial to distinguish malignant tumors from benign ones. Such information is obtained from emission PET images (using 18-FDG as injected tracer), where malignant tumors are visualized as bright and well-contrasted unexpected structures. On the other hand, emission PET images suffer from strong presence of noise and artifacts, preventing a good localization of anatomical structures. Therefore, the combination of these complemen- 
tary images has a significant impact on oncology decisions such as staging, surgery, treatment or therapy planning. This data integration is performed by means of a registration procedure that consists of a spatial alignment aiming to cope with deformations between CT and PET images. These differences are mainly due to the elastic nature of thoracic and abdominal organs, structure movements induced by respiratory and cardiac cycle, patient's position as well as the different physical principle underlying both acquisition techniques. Even though a considerable amount of research has been carried out on the registration problem, the noisy nature and the high presence of artifacts in emission PET images, as well as the complexity of thoracic and abdominal organs and their movements, make the registration of these images a challenging application.

We have proposed in [1] a registration procedure that includes a structure segmentation step where the most significant homologous thoracic and abdominal organs are recognized in both CT and emission PET images. A non-linear registration phase between these segmented structures is used to initialize a refinement phase working with the whole set of grey-level intensities. The work presented in this paper concerns the segmentation of these structures in CT and emission PET images.

A prerequisite for the registration methodology concerns its practical use in clinical routine, which forces us to develop robust and fast image processing algorithms. As registration techniques are already high computational cost procedures, structure segmentation must be performed in almost real-time in order to dedicate most of the time to the alignment computation. Furthermore, if a minimum interaction is desirable in order to improve result robustness, a fast segmentation method is required.

Given the noisy and complex nature of functional images employed in our application, it could be a serious problem if an accurate segmentation were needed, as would be the case if the final result would only rely on the registration of extracted anatomical structures. Even if it is possible to localize several common structures in both image modalities and if their segmentation in CT volumes can be achieved without excessive problems, their recognition in emission PET scans, in an automatic, robust and accurate way, is not a trivial task (the poor emission PET image quality makes difficult to find some structure contours, even visually). However, in our case, segmentation errors will not necessarily be propagated to the final result, as the second intensitybased registration stage will be able to correct them. Thus, we just need to get a reasonably good approximation of the anatomical features visible in both volumes, or, if that were not possible, as many references about them as can be detected. So, instead of accuracy, our main concerns here are the speed and the reliability of the system.

We propose an original hierarchical segmentation procedure based on robust spatial relationships between the targeted structures. The use of these spatial relationships is motivated by their high robustness and low inter-patient variability, unlike other structure characteristics such as shape or grey-level values. Furthermore, using this strategy, we imitate the reasoning of a physician when interpreting a medical image. The segmentation of each structure is performed in two steps: a first stage composed of automatic thresholding and other low level processing operations in a ROI defined by previously segmented objects; and a second stage consisting in refining the result using a 3D deformable model.

The paper is organized as follows. In Section 2, the proposed hierarchical procedure is detailed, and we present the ROI construction for each targeted structure in both image modalities and the first initial segmentation stage. Afterwards, in Section 3, the refinement phase, performed with a deformable model combined with spatial relationships, is described. In Section 4, some final results are shown and discussed, and finally, in Section 5, conclusions are presented.

\section{Hierarchical procedure}

\subsection{Overview}

In the developed hierarchical procedure, the extraction of a given structure is driven by information derived from anatomical objects which are 
simpler to recognize (thus, they have been previously segmented). This information is composed of spatial constraints (directions, exclusions) inferred from the previously segmented structures and expressed by means of Regions Of Interest (ROI) in which the search for new structures will take place. As mentioned above, the segmentation of each structure is performed in two steps in the corresponding ROI: a first stage where a structure-specific combination of low-level image processing techniques provide an initial segmentation; and a second stage in which a $3 \mathrm{D}$ simplex mesh deformable model refines the previous results.

Our registration methodology requires a list of homologous structures that can always be found and recognized in thoracic/abdominal emission PET and CT images in a robust way. Based on discussions with medical experts, we finally chose to segment the following structures: skin, skeleton, lungs, kidneys and liver. Since the skeleton is difficult to extract with enough robustness in emission PET scans, it is only used for the CT image segmentation as a support structure constraining the construction of some ROIs, but does not play any role in the registration procedure.

Segmentation of the targeted anatomical structures is a challenging task, in particular in functional images. The main problem is that, due to the presence of acquisition artifacts and a strong inter-patient variability, information directly provided by the images for a given structure (i.e. its grey-level values) is not robust and stable enough to guide its segmentation. Consequently, other types of information must be used to complement or constrain information provided by the image. A classical way to introduce such prior information is to use atlases or anatomical models. Its use is always open to criticism when working with pathological images, in particular in oncology applications where tumors may modify the morphology of a given structure. Furthermore, the large structure deformations involved in thoracic and abdominal regions would require a previous registration phase between the atlas and the images to segment, thus considerably increasing the computational cost of the algorithm. On the contrary, we have chosen to mathematically model robust spatial relationships that shall provide prior constraints to combine with information from the images.

Spatial relationships have been rarely used in medical image segmentation algorithms. Some exceptions can be found in $[2,3,4]$, where they are employed in brain imaging applications. They take benefit of considering human body as a structured scene, where some relations between organs possess a high degree of robustness and have a low inter-subject variability. Many examples of stable spatial relations can also be found in thoracic and abdominal regions only using basic knowledge on human anatomy: "stomach is placed above kidneys", "lungs are included inside the thoracic cage", "liver is placed under the lungs", "kidneys are closer to liver than to lungs", ... Although such spatial relationships may seem trivial, their addition to an image processing algorithm can considerably help in constraining or rejecting possible solutions. Therefore, the proposed hierarchical procedure classifies the different anatomical structures in a progressive fashion. The extraction of a given structure will be constrained by the information derived from those which are simpler to recognize. An anatomical structure is considered as simple to segment if its associated intensities are fairly homogeneous, if it is well contrasted with respect to neighboring structures and if its morphology, spatial location and relationships are robust and not very variable among different patients.

In general, spatial relations are categorized into three classes: topological, distance and orientation relations [5]. Morphological and symmetry relations are sometimes added as new classes. In our work, topological, orientation and symmetry spatial relations are used as prior constraints. This information is employed to construct Regions Of Interest (ROI) (see Section 2.2) in which the search for new structures will take place. Furthermore, as it is shown in Sections 2.3 and 3, spatial relationships are also used to select structures in the ROI corresponding to a given criterion and to constrain the subsequent deformable model phase.

The proposed hierarchical method requires a specific order for the segmentation of the struc- 


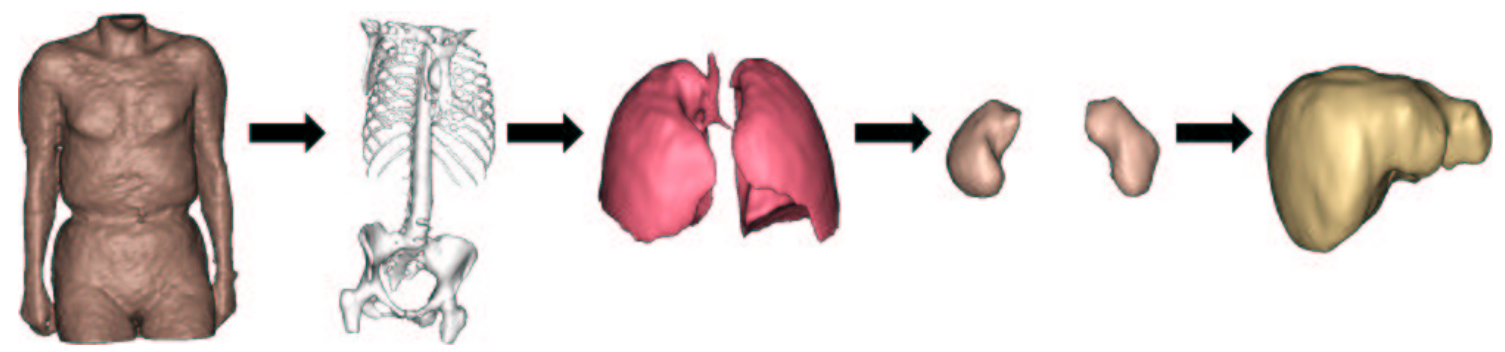

Figure 1. Segmented structures and hierarchical order of the segmentation procedure (from left to right): skin (simplest structure to recognize), skeleton (only in CT images), lungs, kidneys and liver (hardest structure to recognize).

tures. This order, which has been chosen based on structure recognition simplicity and relationship robustness, is (see Figure 1): skin; skeleton (only for CT scan); lungs; kidneys; and liver.

\subsection{Construction of the Regions Of Inter- est (ROI) by means of spatial relation- ships}

As described above, the search of a given structure is limited to a ROI defined using robust and stable spatial relationships with respect to previously segmented structures. Relationships used in the proposed procedure include direction, symmetry and exclusion relations. The ROI for each structure is defined as follows.

\section{Skin and skeleton}

The skin constitutes the first step of the segmentation procedure in $\mathrm{CT}$ and PET images and therefore no ROI is used. Concerning skeleton segmentation in CT scan, it is only constrained to be placed inside the skin volume.

\section{Lungs}

In $\mathrm{CT}$ images, the lung ROI is derived from the skin, i.e., lungs must be included in the area delimited by the skin. In emission PET images ${ }^{3}$ lungs are difficult to recognize. Therefore, we use

\footnotetext{
${ }^{3}$ The majority of PET acquisition protocols produce two PET images: emission and transmission ones. Transmission PET image is only used to correct attenuation in the emission PET one and it is not employed for medical purposes.
}

the transmission PET image, in which lungs are easier to segment in order to obtain the lung ROI for the emission PET image, making the assumption that there are no large lung deformations between emission and transmission scans. The segmentation procedure detailed in Section 2.3 is used to recognize the lungs in the transmission image. Then, we dilate (mathematical morphology dilation) them in order to construct a ROI for the lungs in the emission image.

\section{Kidneys and liver}

In CT images, an upper bound in the $\mathrm{z}$ axis is derived from previously segmented lungs based on the following spatial relationship: "liver and kidneys are under the lungs". This upper limit, computed on each 2D coronal slice, is composed of the lower lung contours and a line linking two automatically extracted landmarks: the lower-left ${ }^{4}$ limit of the right lung (point $B$ in Figure 2) and the lower-right limit of the left lung (point $C$ in Figure 2).

The automatic detection of these landmarks is achieved by the following procedure. For each $2 \mathrm{D}$ coronal slice, the first lung contour point, starting from left columns of the $2 \mathrm{D}$ slice, is selected (point $A$ in Figure 2). Starting from this point, the next landmark is defined as the one which has $\max (x+z), x$ and $z$ being spatial coordinates of the image (point $B$ ). In order to compute the corresponding points in the left lung, the same

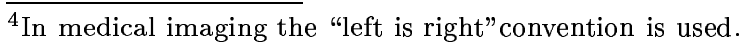



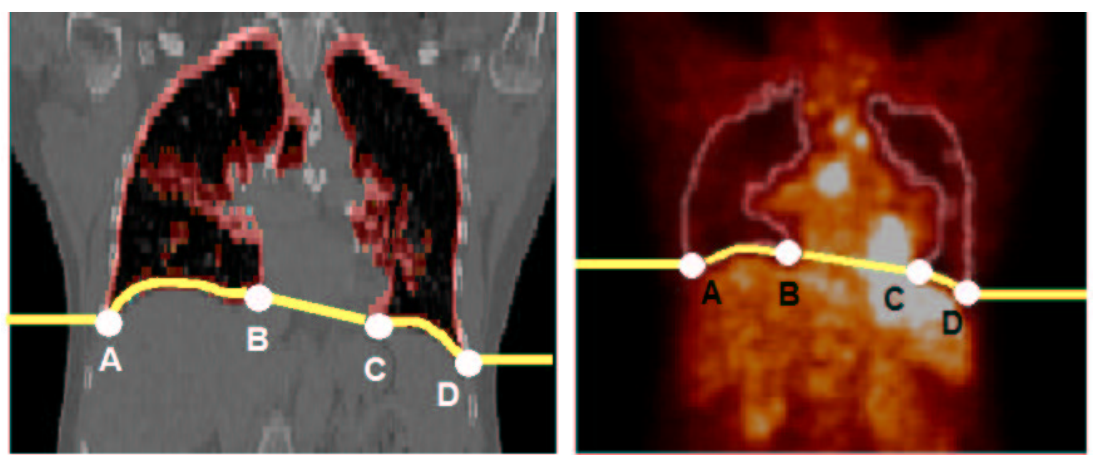

Figure 2. Landmarks (points A, B, C and D) and upper bound obtained from the lungs in CT (left) and emission PET (right) images in order to built liver and kidneys ROI.

procedure is applied in a symmetrical way, this time the second landmark point corresponding to $\max (z-x)$ (point $C$ in Figure 2). Once points $B$ and $C$ are found, a line linking them is automatically drawn, defining, together with the lower lung contours, the upper limit of kidneys and liver ROIs for each 2D coronal slice. This upper bound has proved to be very helpful for separating the liver from the heart, in particular in CT images, where these structures have similar grey-level intensities.

A lower bound is also computed using the top of the pelvis, which is extracted from the skeleton. The top of the pelvis is found by the following procedure. The area of the skeleton surface is computed for each $2 \mathrm{D}$ axial slice. We have observed that this area can be used to detect the first $2 \mathrm{D}$ axial slice in which the spine is visible, coming from the pelvic region in a foot-to-head scanning: the top of the pelvis is obtained when the $2 \mathrm{D}$ axial skeleton area is below a given threshold which is chosen in an experimental way.

Finally, ribs are also used to laterally limit the ROI corresponding to kidneys and liver. First, we remove $z$ slices under the top of the pelvis that has been computed just before. The next step separates the ribs from the rest of the skeleton by computing symmetrical pairs of objects distant from the image symmetry plane in each $2 \mathrm{D}$ coronal slice. Image symmetry plane and selection of symmetrical pairs of objects algorithms are detailed in Section 2.3. Once ribs are found for each $2 \mathrm{D}$ coronal slice, a line linking each rib with its two neighbor ribs is drawn, defining other limits of the ROI, as can be seen in Figure 3.

In PET images, as skeleton is not available, we only use the upper bound derived from segmented lungs.
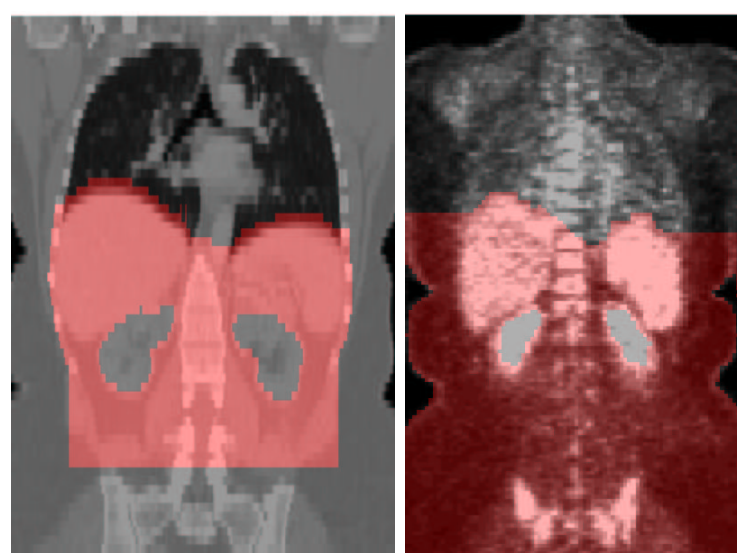

Figure 3. Region of Interest (in red) for liver in CT (left) and PET (right) images.

Concerning the ROI for the liver, in addition the previously segmented kidneys are suppressed 
from the ROI in order to avoid overlap between these structures. An example of liver ROI for both CT and emission PET images is presented in Figure 3.

\subsection{Initial segmentation}

The initial segmentation of each structure is obtained by means of the following sequence of operations (in this order) that are applied in the corresponding ROI:

1. automatic thresholding;

2. binary erosion;

3. selection of connected components;

4. binary dilation;

5. consistency check.

\section{K-means:}

The well-known k-means automatic thresholding method [6] is the first step of the initial segmentation procedure. This method is able to provide satisfactory classification results only if it is applied in a restricted ROI, in which not too many structures (and thus, not too many different grey-level values) appear. Otherwise this method will produce several segmentation errors. This will be typically the case for the liver because it will be classified in the same class as the heart due to the high similarity between their intensities. The use of spatial relationships to construct the ROI solves this problem and allows us to apply automatic thresholding. Nevertheless, an initial phase of parameter tuning (number of classes and their centers) must be performed for each structure. In our case, after this training phase, parameters have proved to be robust enough in order to fix them (for a given anatomical structure) for the whole set of processed images.

\section{Mathematical morphology:}

The two basic binary mathematical morphology operations, erosion and dilation, are used in the initial segmentation procedure. The former, applied after the k-means stage, aims at separating undesirable components from relevant structures and dilation, applied after the selection of connected components stage, recovers some of the data lost in the erosion step.

\section{Selection of connected components:}

This stage consists in selecting the correct connected components among those classified by the automatic thresholding phase as having similar intensities as the targeted structure.

In the case of liver and lung segmentation, the chosen criterion is to select the largest connected components. A noteworthy exception is the case of kidneys because they are not the largest connected components in the ROI. The two most symmetrical components with respect to the body symmetry plane are selected, as illustrated in Figure 4 . The symmetry plane computation is based on an algorithm originally developed for brain images [7]. The search of the symmetry plane is seen as a mono-modal registration problem and it is expressed as the maximization of the following symmetry measure $\sigma_{f}(\Pi)$ :

$$
\sigma_{f}(\Pi)=1-\frac{\left\|f-e_{\Pi}(f)\right\|_{2}^{2}}{2\|f\|_{2}^{2}}
$$

for a 3D gray-level image $f$, where $e_{\Pi}(f)$ is its reflection with respect to a given plane $\Pi$ and $\|f\|_{2}$ denotes the $l_{2}$ metric of $f$.

Once the best reflection symmetry plane is computed, the selection of the most symmetrical components is achieved using a symmetry measure for fuzzy sets, as proposed in [8], which, for a pair of binary objects $(A, B)$ can be formulated as:

$$
S\left(A, e_{\Pi}(B)\right)=\frac{\left|A \cap e_{\Pi}(B)\right|}{\left|A \cup e_{\Pi}(B)\right|}
$$

The pair of connected components having the highest measure will be chosen as the most symmetrical one.

\section{Consistency verification:}

Robustness is a major concern for any medical image processing algorithm. In order to improve the robustness of the initial segmentation procedure (in particular of the steps involving parameter tuning), we automatically verify result consistency after each critical phase. To this purpose 

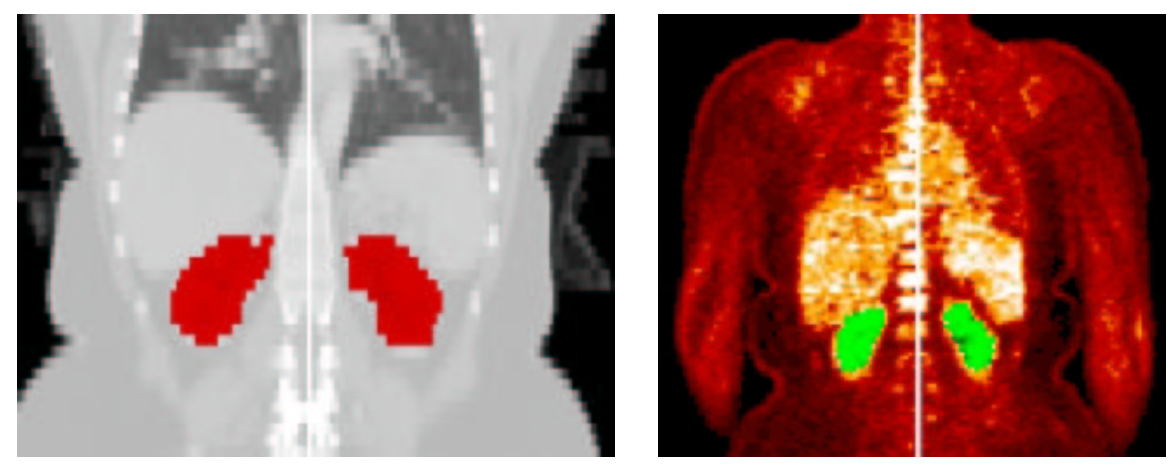

Figure 4. Symmetry plane and kidney symmetry in grey-level CT (left) and PET (right) images

a set of routines have been developed that systematically check for certain parameters of the extracted organ, like dimensions, volume or compactness, to be within the expected range. As a matter of fact, physicians usually ask for an interactive control of structure segmentation procedures, therefore, these check routines will help the development of an user-friendly segmentation interface. From the robustness point of view, the most critical operations include automatic thresholding and selection of connected component stages. Nevertheless, due to the limited size of ROIs where structures are searched, errors produced by these stages have been well identified and taken into account in the procedure.

In each image processing step, when consistency measures are finally within the expected range, the detected region is the input of the following regularization phase.

\section{Refinement with a deformable model}

The previous initial segmentation phase provides a good approximation of the structures, indicating their location in an accurate way. However, this result cannot be considered as an accurate final segmentation result, even in our context of initial registration. The main problem is the lack of regularization. This problem is overcome using a 3D simplex mesh deformable model [9].

Deformable models [10] are curves or surfaces defined in a given image domain which evolve un- der the influence of constraints computed from image data (usually an edge map) and regularity constraints. They have been largely used in several image processing and computer vision applications. Basically, there are two main types of deformable models: parametric [10] and geometric $[11,12]$ ones.

Due to the particular requirements of our application, a parametric technique has been chosen. Geometric deformable models allow the structure topology to change but in our specific problem, this is not needed and is even undesirable, according to the physician's point of view. Furthermore, when parametric deformable models are implemented using a discrete representation, a fast convergence can be obtained. Parametric techniques have also the advantage of naturally allowing the use of initial results (as obtained in the first segmentation phase) and the introduction of heterogeneous prior information.

\subsection{Evolution of the deformation model}

The evolution of the deformable surface $\mathbf{X}$ is described by the following dynamic force equation:

$$
\gamma \frac{\partial \mathbf{X}}{\partial t}=\mathbf{F}_{i n t}(\mathbf{X})+\mathbf{F}_{e x t}(\mathbf{X})
$$

where $\mathbf{F}_{\text {int }}$ is the internal force related to physical properties or constraints of the model that specifies the regularity (elasticity and stiffness) of the surface and $\mathbf{F}_{\text {ext }}$ is the external force that drives 


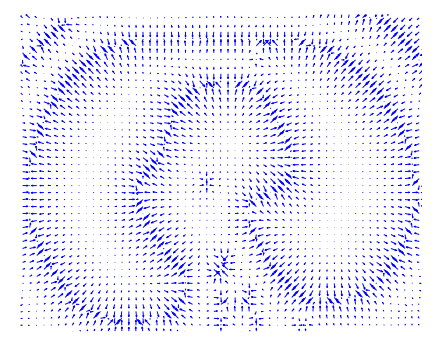

(a)

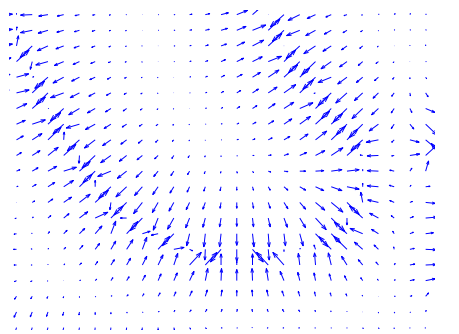

(b)

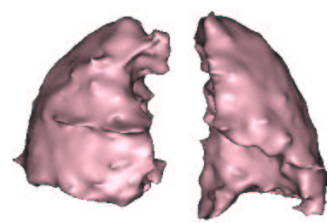

(c)
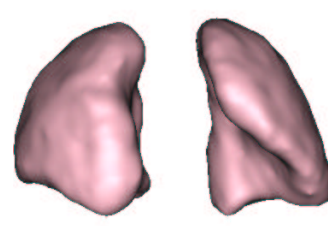

(d)

Figure 5. Axial slice of GVF computed on an emission PET image (a) and detail (b); example of an initial segmentation (c) and its refinement with the deformable model (d).

the surface towards the desired features in the image. The final solution is the steady state of the previous equation. The chosen internal force is:

$$
\mathbf{F}_{\text {int }}=\alpha \nabla^{2} \mathbf{X}-\beta \nabla^{2}\left(\nabla^{2} \mathbf{X}\right)
$$

where $\alpha$ and $\beta$ respectively control surface tension (prevent it from stretching) and rigidity (prevent it from bending) and $\nabla^{2}$ is the Laplacian operator.

\subsubsection{Gradient Vector Flow}

The choice of the external force is crucial in order to make the deformable model evolve towards contours of the object to segment. $\mathrm{Xu}$ et al. [13] have proposed an external force based on the diffusion of the gradient vector associated to a given contour map, called Gradient Vector Flow (GVF), and its generalization [14] assures a large capture range and convergence towards possible contour concavities. The GVF technique produces dense vector fields by solving the equation of vectorial diffusion that distributes gradient vectors of a binary or grey-level contour map computed from the image. The GVF field $u(x)$ of the function $f(x)$ is defined as the equilibrium solution of the following vector diffusion equation:

$$
\left\{\begin{array}{l}
\mathbf{u}_{t}=g(|\nabla f|) \nabla^{2} \mathbf{u}-h(|\nabla f|)(\mathbf{u}-\nabla f) \\
\mathbf{u}(x, 0)=\nabla f(x)
\end{array}\right.
$$

where $u_{t}=\frac{d u}{d t}$ and $t$ is the time parameter.
The function $f(x)$ can be any edge map. Here a classical Canny-Deriche [15] edge detector followed by a hysteresis thresholding is used for computational cost reasons. Functions $g(\cdot)$ and $h(\cdot)$ are weighting functions that apply respectively to smoothing and data terms. They depend on the contour map gradient, which is spatially variable, thus, these functions will also vary through the space domain. As it is desirable that the vector field will vary in a slow and smooth fashion far away from contours (where the gradient is weak), but at the same time will act as $\nabla f$ near them, $g(\cdot)$ and $h(\cdot)$ must be decreasing and increasing functions of $|\nabla f|$, respectively. The following weighting functions accomplish these conditions:

$$
\begin{aligned}
& g(|\nabla f|)=e^{-\left(\frac{|\nabla f|}{K}\right)^{2}} \\
& h(|\nabla f|)=1-g(|\nabla f|),
\end{aligned}
$$

where $\mathrm{K}$ determines the trade-off between the field smoothness and the fidelity to the image contour gradients. An example of a GVF vector field, computed on an emission PET grey-level image, is shown in Figure 5.

\subsubsection{Regions of Interest constraining the deformable model}

In order to prevent the deformable model from progressing beyond the limit of some structures, we propose to introduce prior information derived from spatial relationships between structures, which have already been used in the initial 
segmentation stage, into the deformable model formulation, resulting in a new external force.

An approach for integrating spatial relations into deformable models has been proposed in [4]. This approach deals with relations represented by fuzzy sets which can be seen as fuzzy ROIs. In our case, the ROIs are binary and are therefore considered as a particular case of the previous approach. The integration is performed by means of an additional term inserted in the external force formulation. This term, called a limiting force, should be combined with the one derived from the image data and must constrain the model to prevent it from going outside the ROI. Furthermore, the model must be only driven by the data term within the ROI, thus the limiting force will be zero inside the ROI.

Therefore, the external force is not only derived from image edges but also constrains the deformable model to stay in the ROI. It can be written as a linear combination:

$$
\mathbf{F}_{e x t}=\lambda \mathbf{v}+\mu \mathbf{F}_{R O I}
$$

where $\mathbf{v}$ is the GVF field, $\mathbf{F}_{R O I}$ is the force attached to the ROI and $\lambda$ and $\mu$ are weighting parameters.

The second term of the external force is used to prevent the deformable model from going outside the ROI. This has proved to be very useful for example for separating the liver from the heart. $\mathbf{F}_{R O I}$ is a distance potential force [16] and it can be written as follows:

$$
\mathbf{F}_{R O I}(x)=-\frac{\nabla d(x)}{\|\nabla d(x)\|}
$$

where $d$ is a distance map to the ROI. It should be noted that we also use the ROI as a mask on the GVF and thus the GVF is equal to zero outside the ROI. Finally, we also use the ROI as a mask on the obtained segmentation to ensure that no objects are overlapping.

\subsection{Simplex mesh deformable model}

A discrete representation has to be chosen in order to implement the parametric deformable model to achieve the refinement stage. Simplex meshes, introduced in [9], are the selected discrete representation. This decision avoids prob- lems due to surface parameterization while remaining a reasonable computational cost algorithm. In addition, local shape constraints can be added to the model in an easy way, and there are also simple discretization methods for the evolution equations and topologic and geometric adaptation algorithms. Other good properties of this approach include that they are are topologically dual to triangulations and have a constant vertex connectivity. Complete details on simplex meshes can be found in [9].

The following procedure is used to obtain simplex meshes. First, initial structure segmentations are systematically eroded in order to ensure that this initialization is inside the targeted object. The next step consists in transforming them into a triangulation. This transformation is performed by means of an isosurface algorithm [17] based on tetrahedra. Once the triangulation is computed, the mesh must be decimated in order to reduce the number of triangles to a manageable quantity. The last step converts the triangular mesh into a simplex mesh [9], using the dual operation. The evolution of each vertex of the simplex mesh is controlled by the dynamical equation 2 , which is discretized by means of the finite difference method [18]. An example of the influence of this regularization phase is shown in Figure 5.

\section{Results}

In our work, 20 data sets composed of $\mathrm{CT}$ and PET (emission and transmission) scans of thoracic and/or abdominal regions provided by three different hospitals have been used. Some structure segmentation results in both $\mathrm{CT}$ and emission PET images are shown in Figures 6, 7 and 8. In the first example, simplex meshes of final segmented structures (skeleton, lungs, kidneys and liver) are superimposed on $\mathrm{CT}$ and PET greylevel images. In the second (CT) and third (PET) images, 2D axial slices of original grey-level volumes are superimposed with contours of final segmentation results corresponding to lung, kidney and liver structures.

A quantitative validation of these results is lacking because it would require too much ad- 

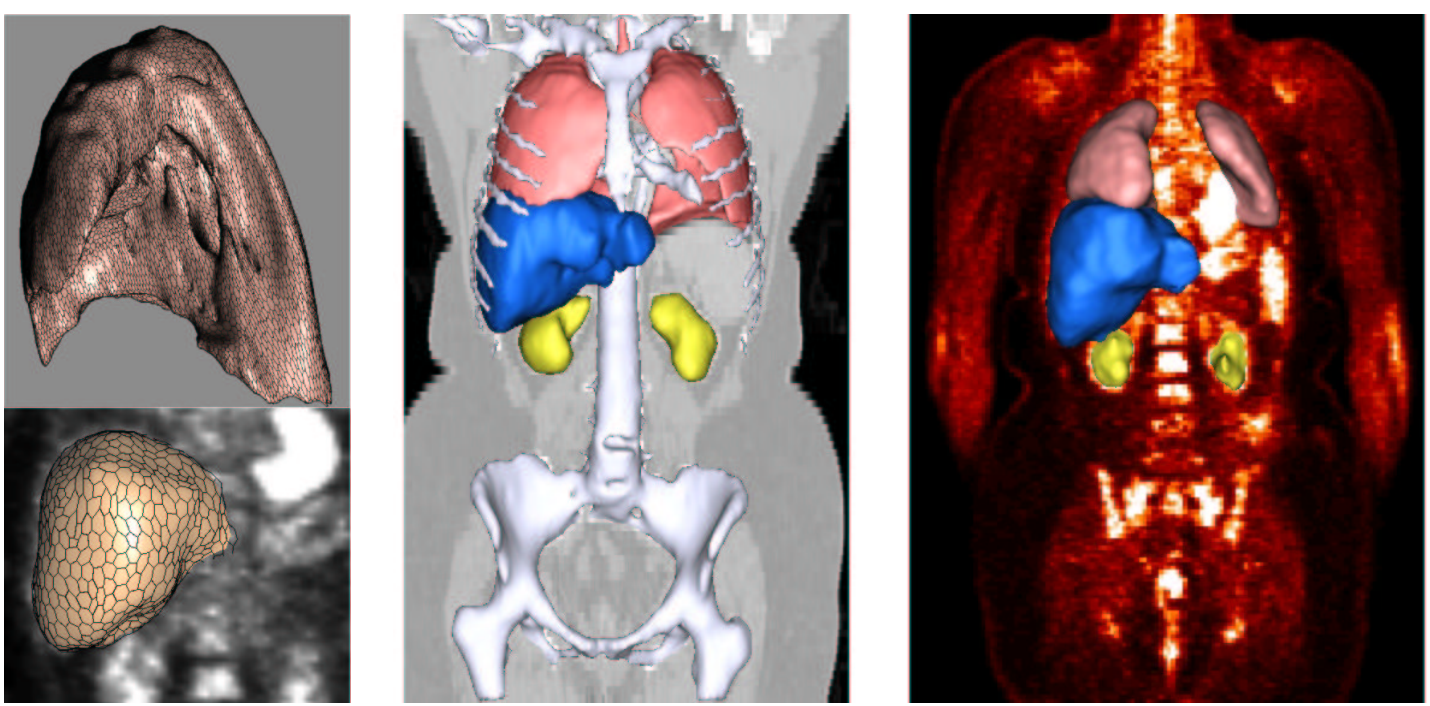

Figure 6. Left: examples of simplex meshes of the segmented structures (top: CT lung; bottom: PET liver). Middle: 3D rendering of CT segmented structures superimposed on a coronal slice. Right: 3D rendering of PET segmented structures superimposed on a coronal slice.

ditional time, since it involves the tedious acquisition of manual ground-truth segmentations (in emission PET images, more than tedious, manual structure segmentations will not be reliable enough to assess a segmentation procedure). Furthermore, the end-goal validation criterion is not the segmentation accuracy, but the final mapping of the anatomy in both data sets.

Nevertheless, we have assessed segmentation results in two different ways: a visual inspection of these results; and a retrospective segmentation evaluation from the assessment of final registration results.

Visual inspection of segmentation results have been done by superimposing them on original grey-level images and comparing corresponding structure contours. The visual accuracy criterion has taken into account our context of initial registration and it should be more severe if these segmented structures would be used as final results. Segmentation results have been positively evaluated by medical experts, in all CT and PET structures. Lung segmentation results are very accurate in CT images (in practice, we obtain global sub-voxel segmentation accuracy) while in PET ones, some insignificant errors (these errors do not induce further misregistrations) appear due to the low SNR of these images. Concerning kidney segmentation, the symmetry plane procedure has proved to be very efficient and the only problem has been the classification of the inner part of these structures, that sometimes has different grey-level values. A 3D hole filling processing stage has solved this situation. The liver is the most difficult structure to segment both in CT and PET images due to the high presence of neighboring structures with similar greylevel values. A reasonable approximation of the liver is obtained, notably separating it from kidneys and heart. Nevertheless, we have sometimes found unidentified small structures close to the liver that our procedure does not separate. In our application, such situation is not a major concern but in other medical segmentation applications it must be classified as a bad segmentation result.

The second way of evaluating segmentation result accuracy is based on the assessment of final registration results obtained by the proposed 


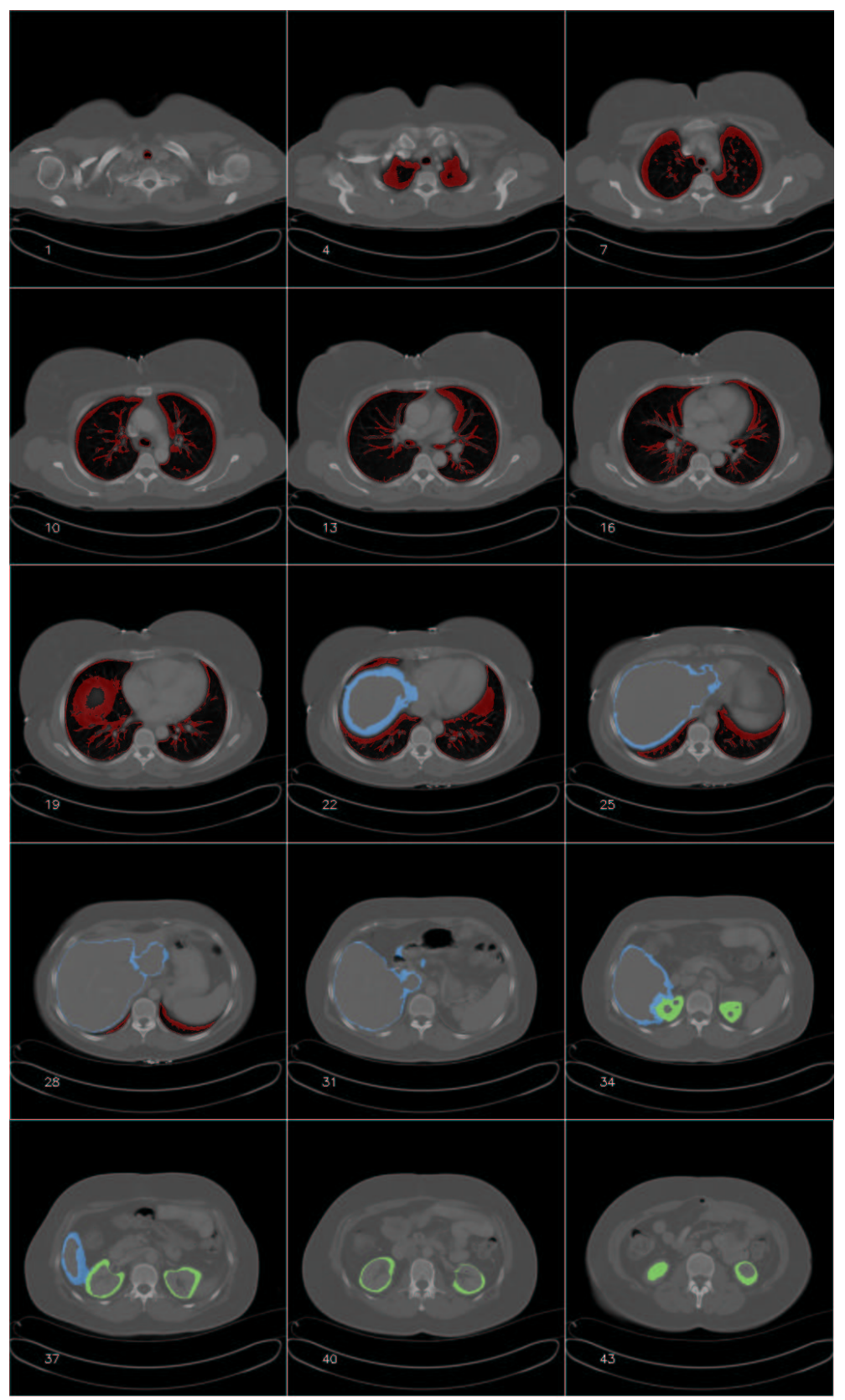

Figure 7. CT axial slices of final segmentation results. 


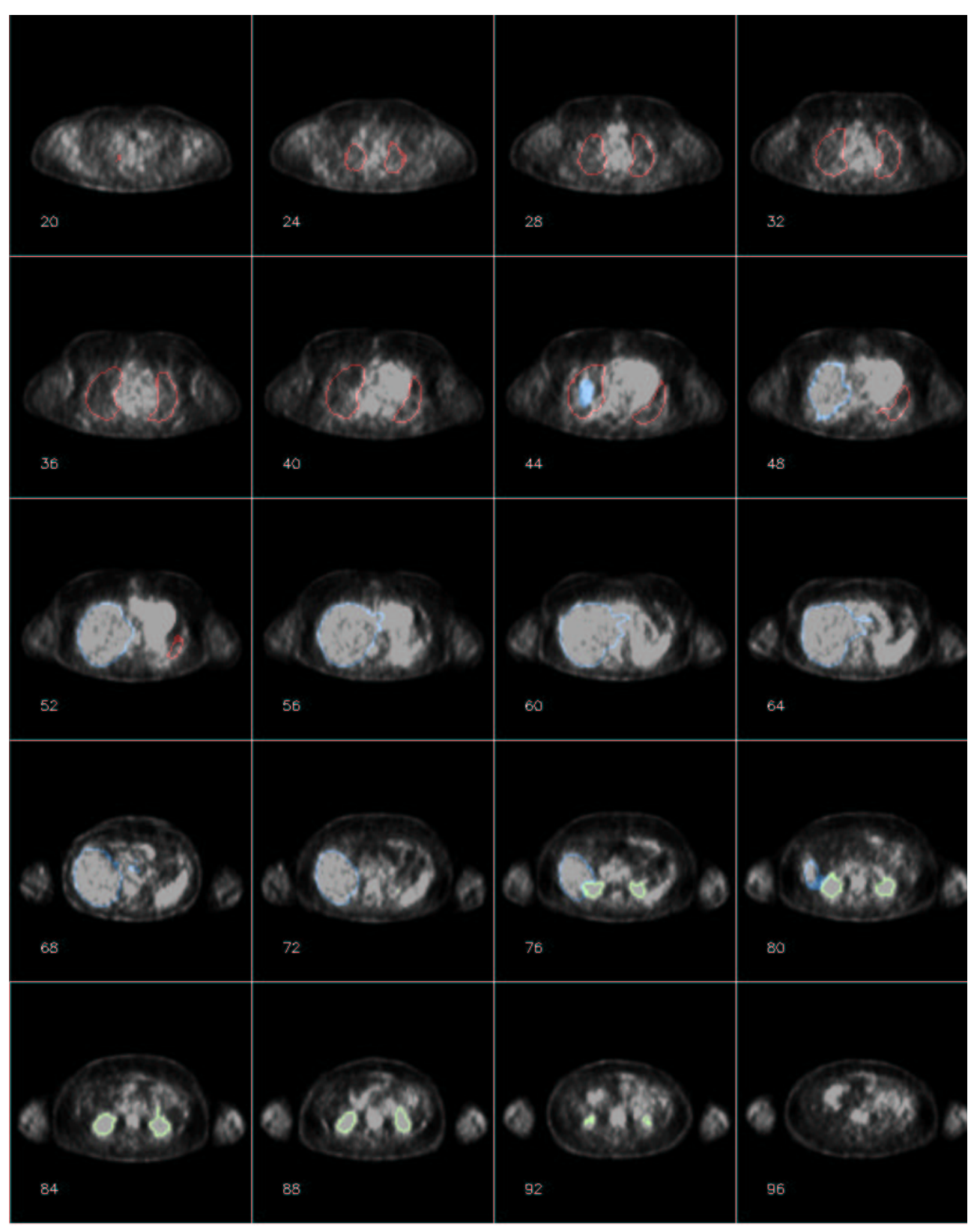

Figure 8. PET axial slices of final segmentation results. 
methodology. As a matter of fact, the transformation computation between CT and PET images strongly depends on the anatomical information that we are capable to extract from both data volumes, i.e., it depends on the segmentation result accuracy. Therefore, the evaluation of final registration results can also be seen as a retrospective assessment of the segmentation stage. We have developed a non-linear registration evaluation protocol [19] that provides a semi-quantitative measure of the registration accuracy. Mean detected registration errors are lower than $1 \mathrm{~cm}$ (thus, within the acceptable error range) for the majority of the evaluated regions including lungs, kidneys, liver and heart. On the other hand, some significant misregistrations appear near the stomach. These data illustrate the fact that registration is better achieved around structures that have been recognized with the segmentation procedure detailed in this paper.

\section{Conclusions}

In this paper, we have presented an original hierarchical segmentation procedure aiming at segmenting several significant anatomical structures (lungs, kidneys, liver) in CT and emission PET images. The segmentation strategy has been implemented according to the special characteristics of the structures, the images involved in the application and our context of initial registration. For each particular structure, specific prior information has been used that can not be generalized to other structures or even to the same structures imaged with other modalities.

The main concerns in this application has been robustness and convergence speed and the proposed segmentation procedure has been designed in order to satisfy them. Concerning robustness, consistency check routines have been added after segmentation steps involving less robust parameter tuning. With respect to convergence speed, the procedure employs low computational cost algorithms in the initial segmentation phase, while the regularization phase has been implemented to assure a fast convergence as well. For a pair of images with standard dimensions (CT image with a size of $512 \times 512 \times 60$ voxels and a PET image with $144 \times 144 \times 200$ voxels), the initial segmentation phase takes about 60 seconds and the regularization phase about 90 seconds for each structure. These computation times fulfill time constraints of our algorithm, thus having a larger time margin for registration algorithms of the proposed methodology.

The use of a hierarchical procedure takes advantage of stable spatial relationships between structures to construct the ROIs where the search of a given structure takes place. The ROI construction is very structure-specific because it is based on spatial relationships which can be valid only for a given anatomical feature. In this way, segmentation of the most difficult structures, such as liver in emission PET scans, has been achieved by using classical image processing techniques that could not been used without spatial constraints provided by simpler and previously segmented structures.

Using this hierarchical procedure, a good tradeoff between speed and segmentation accuracy has been achieved. An important drawback of this strategy could be the error propagation between different segmentation stages, i.e., errors obtained in the segmentation of simpler structures will highly influence the segmentation of subsequent structures. Here again, the consistency checks included in the procedure appear to be very important and allow us to avoid such problems.

Final segmentation results have proved to be accurate enough to guide the structure registration procedure that will initialize, close to the final solution near segmented structures, the refinement registration step working with the whole set of intensities. A visual inspection of the results has been done because a quantitative validation would ask for a tedious manual segmentation of several structures in CT and emission PET images. Furthermore, such manual segmentations will not be reliable enough for segmented structures in emission PET images due to their low SNR. Moreover it has been shown that the proposed segmentation leads to very accurate registration results. Future work will focus on the addition of other structures such as the heart and the stomach to the hierarchical segmentation procedure. 


\section{Acknowledgments}

The authors would like to thank Dr. Hervé Foehrenbach, Dr. Pierre Rigo, Dr. Xavier Marchandise, Gaspar Delso, the research team of S.H.F.J. in Orsay and the members of Segami Corporation for their contribution to this project. This work was partially supported by the French Ministry for Research (grant number 01B0267).

\section{References}

[1] O. Camara, G. Delso, and I. Bloch. Free Form Deformations guided by Gradient Vector Flow: A surface registration method in thoracic and abdominal PET-CT applications. In Workshop in Biomedical Image Registration (WBIR'03), pages 224-233, Philadelphia, PA, USA, 2003.

[2] I. Bloch, T. Géraud, and H. Maître. Representation and fusion of heterogeneous fuzzy information in the 3D space for model-based structural recognition - application to $3 \mathrm{D}$ brain imaging. Artificial Intelligence Journal, 148:141-175, 2003.

[3] A. Pitiot, H. Delingette, N. Ayache, and P.M. Thompson. Expert knowledge guided segmentation system for brain MRI. In International Conference on Medical Image Computing and Computer-Assisted Intervention (MICCAI'03), pages 644-652, Montreal, Canada, 2003.

[4] O. Colliot, O. Camara, R. Dewynter, and I. Bloch. Description of brain internal structures by means of spatial relations for MR image segmentation. In SPIE Conference on Medical Imaging: image processing, Bellingham, WA, USA, 2004.

[5] B.J. Kuipers and T.S. Levitt. Navigation and Mapping in Large-Scale Space. Artificial Intelligence Magazine, 9(2):25-43, 1988.

[6] R. Duda, P. E. Hart, and D. G. Stork. Pattern Classification. Wiley, New York, USA, 2nd edition, 2001.
[7] A.V. Tuzikov, O. Colliot, and I. Bloch. Evaluation of the symmetry plane in 3D MR brain images. Pattern Recognition Letters, 24(14):2219-2233, oct 2003.

[8] O. Colliot, A.V. Tuzikov, R.M. Cesar, and I. Bloch. Approximate Reflectional Symmetries of Fuzzy Objects with an Application in Model-Based Object Recognition. Fuzzy Sets and Systems, 2004. in press.

[9] H. Delingette. General object reconstruction based on simplex meshes. International Journal of Computer Vision, 32(2):111-146, 1999.

[10] M. Kass, A. Witkin, and D. Terzopoulos. Snakes: Active Contour Models. International Journal of Computer Vision, 1(4):321-331, 1987.

[11] R. Malladi, J.A. Sethian, and B.C. Vemuri. Shape modelling with front-propagation: a level set approach. IEEE Transactions on Pattern Analysis and Machine Intelligence, 17(2):158-175, 1995.

[12] V. Caselles, R. Kimmel, and G. Sapiro. Geodesic active contours. In International Conference on Computer Vision (ICCV'95), pages 694-699, Cambridge, MA, USA, 1995.

[13] C. Xu and J.L. Prince. Snakes, shapes and gradient vector flow. IEEE Transactions on Image Processing, pages 359-369, March 1998.

[14] C. Xu and J.-P. Prince. Generalized gradient vector flow external forces for active contours. Signal Processing, An International Journal, 71(2):131-139, December 1998.

[15] R. Deriche. Using Canny's criteria to derive a recursively implemented optimal edge detector. International Journal of Computer Vision, 1(2):167-187, 1987.

[16] L.D. Cohen and I. Cohen. Finite Element Methods for Active Contour Models and balloons for 2D and 3D images. IEEE Transactions on Pattern Analysis and Machine Intelligence, 15(11):1131-1147, 1993. 
[17] B. Piquet, C. T. Silva, and A.E. Kaufman. Tetra-Cubes: An algorithm to generate 3D isosurfaces based upon tetrahedra. In Brazilian Symposium on Computer Graphics and Image Processing (SIBGRAPI'96), volume 21, pages 205-210, Caxambu, Brazil, 1996.

[18] C. Xu. Deformable Models with Application to Human Cerebral Cortex Reconstruction in Magnetic Resonance Images. $\mathrm{PhD}$ thesis, Johns Hopkins University, 2000.

[19] O. Camara. Non-linear registration of thoracic and abdominal $C T$ and PET images: methodological study and application in clinical routine. PhD thesis, Telecom Paris, 2003. 